DOI: https://doi.org/10.46296/rc.v3i6.0016

\title{
Comportamiento de compra y productos de bioseguridad durante la pandemia
}

\section{Purchase behavior and biosecurity products during the pandemic}

\author{
Autor: Villegas-Macías Fidel Alejandro \\ Universidad Laica Eloy Alfaro de Manabí (ULEAM). Manta, Ecuador. \\ e1350516021@live.uleam.edu.ec
}

\begin{abstract}
RESUMEN
En la actualidad el mundo ha presentado un gran giro dentro de sus comportamientos comerciales y adquisitivos, puesto que en el contexto de productos e insumos cómo equipos de protección personal, los cuales anteriormente sólo eran utilizados en ciertos sectores muy puntuales, tales como en ámbitos industriales, medicina, entre otros, puesto que preservan la bioseguridad de una persona, han sido adoptados como productos esenciales que conforman las bases de la denominada nueva normalidad originada a partir de la pandemia del covid-19. por eso analizar el comportamiento de las personas al momento de comprar estos productos de bioseguridad es uno de los puntos más importantes, además de comprender cómo esta demanda crea un nuevo mercado y examinar si se están adquiriendo los productos con una calidad específica para los mismos o si sólo se está adquiriendo productos por una demanda que es impulsada por la crisis sanitaria vigente.
\end{abstract}

Palabras Clave: Pandemia, Equipos de protección personal, productos de bioseguridad, consumismo en épocas de Covid-19.

\begin{abstract}
At present the world has presented a great turn within its commercial and purchasing behavior, since in the context of products and supplies such as personal protective equipment, which previously were only used in certain very specific sectors, such as in industrial areas, medicine, among others, since they preserve the biosecurity of a person, they have been adopted as essential products that form the basis of the socalled new normal originated from the covid-19 pandemic. That is why analyzing the behavior of people when buying these biosafety products is one of the most important points, in addition to understanding how this demand creates a new market and examining whether they are acquiring products with a specific quality for them or if you are only purchasing products due to a demand that is driven by the current health crisis.
\end{abstract}

Keywords: Pandemic, personal protective equipment, biosecurity products, consumerism in times of Covid-19.

Información del manuscrito:

Fecha de recepción: 19 de mayo de 2020.

Fecha de aceptación: 09 de julio de 2020.

Fecha de publicación: 10 de julio de 2020. 


\section{INTRODUCCIÓN}

En el presente trabajo se van a tratar temáticas contemporáneas, muchas de conocimientos generales por el tema de la emergencia sanitaria en un estado histórico y muy cambiante como lo es una pandemia y cómo la misma ha desencadenado en la alteración del comportamiento de los diferentes individuos que producen y adquieren productos, al momento de comprar; la presencia de los insumos de bioseguridad, además de diferentes productos de equipos de protección personal durante esta emergencia sanitaria a nivel mundial y sus implicaciones socioeconómicas dentro de las diferentes industrias, Asimismo como se ha trasformado el precio de los diferentes productos y servicios por la presencia del Covid-19, las diferentes actividades comerciales y cómo afecta esto a la economía mundial y Por ende Cuáles serían estas tendencias que impulsaba el cambio dentro de la economía y los nuevos estilos de consumismo

Teniendo en cuenta lo que es la seguridad alimentaria, Ya que en este tiempo se ven afectadas las Industrias de las cuales dependen de la importación y la exportación y sumándole a esto la dificultad para obtener suministros locales, alimentos que también son algo trascendental para la comercialización y la economía, ya que si estas presentan interrupciones de diferentes actividades que técnicamente son básicas para mantener un equilibrio dentro de la economía de diferentes territorios, pero todo esto antes mencionado son cosas que van a generar un costo dentro de los diferentes productos a realizarse y Por ende va haber una elevación de algunos y en otros una caída por la demanda muy baja que presenta, $\mathrm{Y}$ así vamos a ir desglosando temas que están presentes dentro de comportamiento de las personas al momento de comprar productos de necesidades básicas y como estos productos de necesidades básicas también han evolucionado para la subsistencia de los mismos.

\section{METODOLOGÍA}

Los fenómenos grandes como las pandemias que se han presentado a nivel mundial son un tema muy delicado de tratar no sólo teóricamente sino también en la práctica y eso ha sido demostrado a lo largo de los años con un sin número de actividades que las personas han realizado para poder 
contenerlas, en la historia se pudo contemplar cómo estás puede repercutir en la salud de los seres humanos y cómo afecta el bienestar económico del mundo entero y su estabilidad, cambiando por completo al mundo de cómo lo conocíamos antes dé, el tema con las pandemias de gripe es que estas son mucho más volátiles qué otras pandemias que sean por algún tipo de contaminación diferente, Por ende se pudo encontrar un sin número de organizaciones a nivel mundial que se encargan de la mitigación, del control de la seguridad y de preservar la seguridad y saludo de las persona, Pero uno del más importantes y reconocidos a nivel mundial es la Organización Mundial de la Salud o también conocida como por sus siglas como la OMS la cual ha tenido una presencia considerable por algunos años a nivel mundial sobre diferentes fenómenos recurrentes en la salud de las personas, en el 2009 se actualizó estas guías de las OMS para el tema de las pandemias de gripe por la presencia del virus $A(H 1 N 1)$ que afectó a muchas partes del mundo, causando que un sin número de personas fallecieran por el mismo, también se tuvo la presencia de otras pandemias que no llegaron a ser tan globales, pero sí tuvieron un gran impacto en el lugar donde desenvolvieron, como lo fue: "Las epidemias de síndrome respiratorio agudo grave (SARS, por sus siglas en inglés) y síndrome respiratorio de Medio Oriente (MERS), Originada también en China, en noviembre de 2002, la epidemia de SARS eventualmente se extendió por 26 países, con 8.098 casos confirmados y 774 muertos. Pero como destaca la profesora Annelies Wilder-Smith, de la Escuela de Higiene y Medicina Tropical de Londres, "para julio de 2003 ya había sido controlada, en un plazo de ocho meses".

El primer brote de MERS, por su parte, fue registrado en Arabia Saudita en abril de 2012. Y aunque a diferencia del SARS-CoV el MERS-CoV todavía no ha podido ser erradicado, más de ocho años después el número de casos confirmados es de nada más 2.494, en 27 países. Además, según los últimos datos de la Organización Mundial de la Salud, los muertos por MERS solamente suman 858." (Wallace, 2020)

Ambas pandemias procedentes del coronavirus presente en animales, claro está que el covid-19 o SARS-CoV-2 ha tenido un desenvolvimiento en las 
personas con afectaciones mucho más drásticas, de las cuales se pudo constatar no solo en la cifra de muertos a nivel mundial, esto nos dejó a uno de los temas puntuales que queríamos llegar.

\subsection{Consumismo en época de pandemias}

"Tomando en cuenta la temporalidad, y dado que el brote de la influenza se dio previo a mayo, históricamente considerado un buen mes para los autoservicios por la celebración del "Día de las madres", ese año se rompió con la buena racha para este tipo de establecimiento.

Los especialistas esperan que, para esta primera etapa, en la que se están realizando compras de pánico, que los establecimientos como autoservicios deberían estar bien surtidos, aunque si habrá una baja en las visitas, ya que la gente no está dispuesta a salir, por lo que en este periodo el comercio electrónico tomará relevancia como sucedió en China, donde el efecto del coronavirus impulsó al e-commerce.

Lo que evidenció la epidemia del 2003, y las crisis de 2008 y 2009 es que todo tiende a normalizarse, tras una contingencia. Pero para eso las marcas deben ser empáticas y contribuir a la sociedad más allá de su Core de negocio. En México, por ejemplo, las plataformas de entretenimiento por streaming, de educación gratuita en línea o los canales de comercio electrónico tienen una gran oportunidad para marcar la diferencia y ofrecer propuestas de valor que contribuyan a un mejor optimismo social.

El ingreso disponible puede estar limitado, pero eso no significa que el comportamiento del consumidor lo lleve a elegir cualquier producto o servicio. Por ello las marcas generar confianza y afinidad, pues las personas necesitan que las entiendan desde su propia personalidad y necesidades emocionales, funcionales y económicas.

Las marcas están obligadas a actuar y a cambiar con la gente, de manera correcta y sin miedo. El entendimiento tanto de su consumidor como de la situación actual es fundamental, ya que tendrán que escoger muy bien, bajo su ADN, qué realizarán, cuándo y cómo. 
Las predicciones financieras de la empresa JP Morgan indican claramente un descenso abrupto en el PIB global, regional y local, a causa del Covid-19. Para Latinoamérica, se estima una caída de 1,2\% en este primer trimestre, $11,6 \%$ en el próximo y $1,2 \%$ en el acumulado anual. Pero el estudio antes mencionado refiere que las marcas más fuertes se recuperan 9 veces más rápido luego de una crisis o situación coyuntural que no podemos controlar." (Kantar, 2020).

\subsection{Implicaciones Socioeconómicas}

"A diferencia de lo que ocurre en el caso de los desastres naturales, donde las disrupciones experimentadas en la provisión de servicios a/de las empresas derivan del daño a las infraestructuras, en el caso de una pandemia estarán principalmente relacionadas con los recursos humanos.

Las consecuencias específicas que la pandemia tendrá en cada empresa dependerán del impacto directo de la pandemia en la salud de los trabajadores y del impacto que tengan las medidas puestas en marcha para hacer frente a la pandemia de gripe dentro del Plan de Continuidad de la empresa. Así, la organización que las empresas hayan sido capaces de desarrollar será fundamental para garantizar que los núcleos clave de su actividad siguen funcionando. Por ello, en el Plan de Continuidad frente a una pandemia de gripe de cada empresa deberán definirse en detalle aquellas funciones que se consideren esenciales dentro de la empresa y que son absolutamente necesarias para mantener la productividad en niveles aceptables durante la pandemia, identificando y cuantificando el personal y actividades imprescindibles. El Plan de Continuidad deberá incorporar también, las soluciones previstas con los actuales recursos disponibles dentro de la empresa." (consumo, 2007 )

\subsection{Precios y Covid}

"En marzo, cuando se presentaron los primeros contagios y comenzaron los rumores sobre medidas de aislamiento social y cuarentena, las compras nerviosas dominaron el comportamiento de los consumidores y las tiendas de barrio se convirtieron en las reinas del abastecimiento. 
Pero a medida que el confinamiento se endureció y se conocieron las reglas de juego de una movilidad limitada al abastecimiento básico, los cambios se fueron notando. Los supermercados ganaron relevancia y participación en el bolsillo de los consumidores, dispuestos a comprar un mayor volumen para no tener que hacer tantas salidas de su hogar. El e-commerce también se convirtió en el canal amigable que muchos no pensaban que era.

Los precios, que antes de la pandemia mandaban en la decisión de compra, también quedaron relegados. La consigna era estar muy bien provisionados. Con el paso de los días, los consumidores han ganado confianza y la apertura gradual, así como el escenario poscovid, implica nuevos cambios.

Carolina Vargas, experta en temas de precios (pricing expert) en la empresa de investigación de mercados Nielsen, explica que en marzo las compras de los colombianos priorizaron productos como alimentos, bebidas y cuidado del hogar, que atendían necesidades básicas como mantenerse limpio, saludable y alimentado.

Uno de los temas llamativos en la pandemia es el de precios y promociones.

Por años, estos fueron la clave del consumo en muchos hogares colombianos, al punto de que la industria y el comercio habían volcado sus esfuerzos en lograr un equilibrio para cautivar clientes. Eso sí, confirmaron que muchos fueron infieles por precios.

Pero la pandemia cambió ciertos paradigmas, porque el desabastecimiento de algunos productos hizo que los consumidores estuvieran dispuestos a pagar más y sin chistar. Pero, al mismo tiempo, muchos empresarios enfrentaron las volatilidades del mercado cambiario y tuvieron que comprar materias primas más costosas, que terminaron reflejadas en los precios.

Estudios de comportamiento de canastas de consumo de Nielsen, empresa que dirige Juan Felipe Saavedra, muestran que las categorías que más crecieron también registran mayor aumento en precios: $20 \%$ en desinfectantes y $12 \%$ en alcohol antiséptico. 
El segundo factor que incide es la ley de oferta y demanda, que rompe paradigmas en algunas categorías como el papel higiénico, uno de los productos más demandados ante el temor de desabastecimiento. Así, muchos consumidores se muestran dispuestos a pagar lo que les pidan.

La devaluación es el tercer tema. En épocas de incertidumbre se ha disparado la cotización del dólar, obligando a importadores y productores a comprar sus materias primas a precios altos, que luego se ven reflejados en sus precios. En el comercio, se estima que $22 \%$ de productos son importados, pero de los restantes muchos usan materias primas compradas en el exterior." (GANDINI, 2020)

La cuarentena nos demostró Cómo puede hacer que la economía y la estabilidad social se vean afectadas por el aislamiento y causar este nerviosismo que domine a los consumidores a tal punto que se creó un caos dentro del comercio para adquirir productos de necesidades básicas y esto a su vez en ciertos puntos hace que para algunas personas de Gran astucia se quieran favorecerse de estos consumidores, y Crean un precio elevado al inicio de estos tiempos no favorecedores, Claro está que después de un tiempo de tener la presencia de virus dentro de nuestro medio, por muchas situaciones de seguridad, bioseguridad, de precautelar la salud, tanto de comprador, como de vendedor, se implementaron numerosos medios para poder mitigar y controlar la propagación del virus, como ya se ha conocido antes, con equipos de protección personal y algunos de bioseguridad que se están dando en estos lugares de compras presenciales, esto genera un costo que por ende se va a haber representado en el precio total del producto adquirir o los diferentes productos 0 servicios.

\subsection{Bioseguridad}

“El 24 de marzo de 2020, la Organización Mundial de la Salud advirtió que "la escasez mundial crónica de equipo de protección personal es una de las amenazas más urgentes para los esfuerzos de contención de virus" en la actual pandemia de enfermedad por coronavirus (COVID-19). En respuesta, se han tomado medidas. tomadas por agencias e instituciones para conservar los suministros actuales al intentar adquirir más equipo de protección personal 
(PPE). Las estrategias de conservación a menudo presentan recomendaciones que sugieren limitar el EPP de nivel superior (por ejemplo, máscaras N95) a ciertas situaciones clínicas. Durante una crisis de salud, los encargados de formular políticas no pueden esperar a que la certeza científica tome decisiones. Por el contrario, requieren enfoques alternativos para enfrentar la incertidumbre y mitigar el riesgo en la toma de decisiones. En este documento, nuestro objetivo es apoyar a los responsables de la toma de decisiones examinando áreas de incertidumbre con respecto a COVID-19, revisando las lecciones de pandemias anteriores (incluido el SARS) y describiendo cómo se puede emplear el principio de precaución en el desarrollo de orientación sobre EPP hasta evidencia científica más sólida se vuelve disponible." (Julieta, Aplicación del principio de precaución a la orientación del equipo de protección personal (EPP) durante la pandemia de \#COVID19, 2020)

"Creación y fortalecimiento de hábitos de higiene (formas adecuadas de estornudar y toser, lavado de manos, manipulación de Equipos de Protección Personal (EPP), cambio frecuente y lavado de la ropa).

Entrega y uso de los EPP necesarios y adecuados, según el puesto de trabajo (protección de ojos, nariz, boca, manos, piel, pies y calzado).

- Protección ocular (gafas, lentes, espejuelos, caretas).

- Boca y boca (mascarilla, tapaboca, nasobuco, mascara).

- Manos (guantes).

- Piel (ropa especial, uniforme, bata, gabacha, delantal).

- Pies y calzado (calzado especial, botas cubre calzado).

- Distanciamiento o aislamiento, manteniendo las distancias de seguridad

- recomendadas entre personas (entre 1,5 y 2 metros)." (Social., 2020)

\subsection{Corrupción consumista}

"En países como Ecuador, México y Colombia fueron reportadas irregularidades y casos de corrupción entre marzo y abril en procesos de adquisición de ventiladores respiratorios, bolsas para cadáveres, ambulancias y diversos insumos de bioseguridad para hacerle frente a la propagación del Covid-19. 
Un negocio redondo y oscuro es el que tienen bajo la lupa las autoridades de países latinoamericanos como México, Ecuador y Colombia por cuenta de la suerte de mina de oro en la que se convirtieron, para algunos funcionarios y empresarios, los contratos de compra de los equipos médicos necesarios para hacerle frente a la propagación del Covid-19.

Ventiladores clínicos que triplican su valor convencional, bolsas para cadáveres a precios exorbitantes, alcohol cobrado como si fuera vino de las más finas reservas y overoles de bioseguridad tan caros como prendas de diseñador, son apenas algunos de los casos reportados en varias naciones de la región en la que la emergencia sanitaria es aprovechada por quienes incrementan gastos a su favor al mismo ritmo con el que aumentan los casos de contagio." (Angulo, 2020).

Los productos de bioseguridad y equipos de protección personal (EPP) se convirtieron en un punto fundamental de comercio y adquisición para los diferentes ciudadanos a nivel mundial, estos han generado en el mercado un cambio en sus formas de posicionamiento de diferentes productos de consumo regulares y encontramos la presencia de alcoholes, desinfectantes, entre otros, cómo productos que se están posicionando básicos en este año y por ende tiene toda relación con la pandemia, además también tenemos otros productos como son las mascarillas, los guantes, gafas y así otros sin números de equipos de protección personal individual, usados para diferentes puntos de comercios, como lo son la industria, médicamente o simplemente para evitar la contaminación de persona a persona al momento de salir de sus hogares por algún motivo diferente a las antes ya mencionado. 


\section{RESULTADOS}

Por medio de la siguiente investigación se pudo recabar información sobre cuál el comportamiento actual que tiene diferentes personas al momento de adquirir productos en esta situación tan nueva y complicada como lo es la de la pandemia de Covid-19, en donde se pudo encontrar que los diferentes comercios y personas que acuden a ellos para que la adquisición de estos productos, han cambiado su forma de distribuir los mismos o brindar un producto un servicio en diferentes formas, precautelando la salud y el bienestar de las personas, además que también para evitar contratiempos con agentes gubernamentales que se tengan en cada país, Esto con respecto a los comercios y la forma de vender sus productos o servicios, pero para las personas que adquieren estos productos también se ve el cambio en su adaptabilidad, para la adquisición de los mismos y el uso de nuevos productos que se convirtieron en básicos, como lo serían los desinfectantes, alcohol, cloro, entre otros De la misma rama de bioseguridad y equipos de protección personal en el caso de mascarillas, gafas, guantes, trajes anti fluidos, entre otras, cosas que muchas veces sólo se las veía en actividades puntuales como temas industriales, de la producción de algún contaminante o manipulación de los mismos, o en temas médicos, Pero estas son cosas que como toda la vida se vio una evolución significativa para el comercio y la economía.

Además, que se analizó por diferentes figuras como lo es en el caso de la figura 3.1.1 en donde nos muestra en comparación con un año anterior que sería el 2018 al año actual en el que estamos 2020 como diferentes actividades que eran cotidianas e incluso a veces no muy comúnmente usadas a diario, Se volvieron más recurrentes por el tema de la pandemia, Asimismo con el Cambio de hábitos y las Reformas en las actividades del comportamiento de diferentes personas. 


\subsection{Figuras y tablas}

Figura 1. Comparación entre las medidas de virus de invierno 2018 y enfermedades infecciosas 2020.

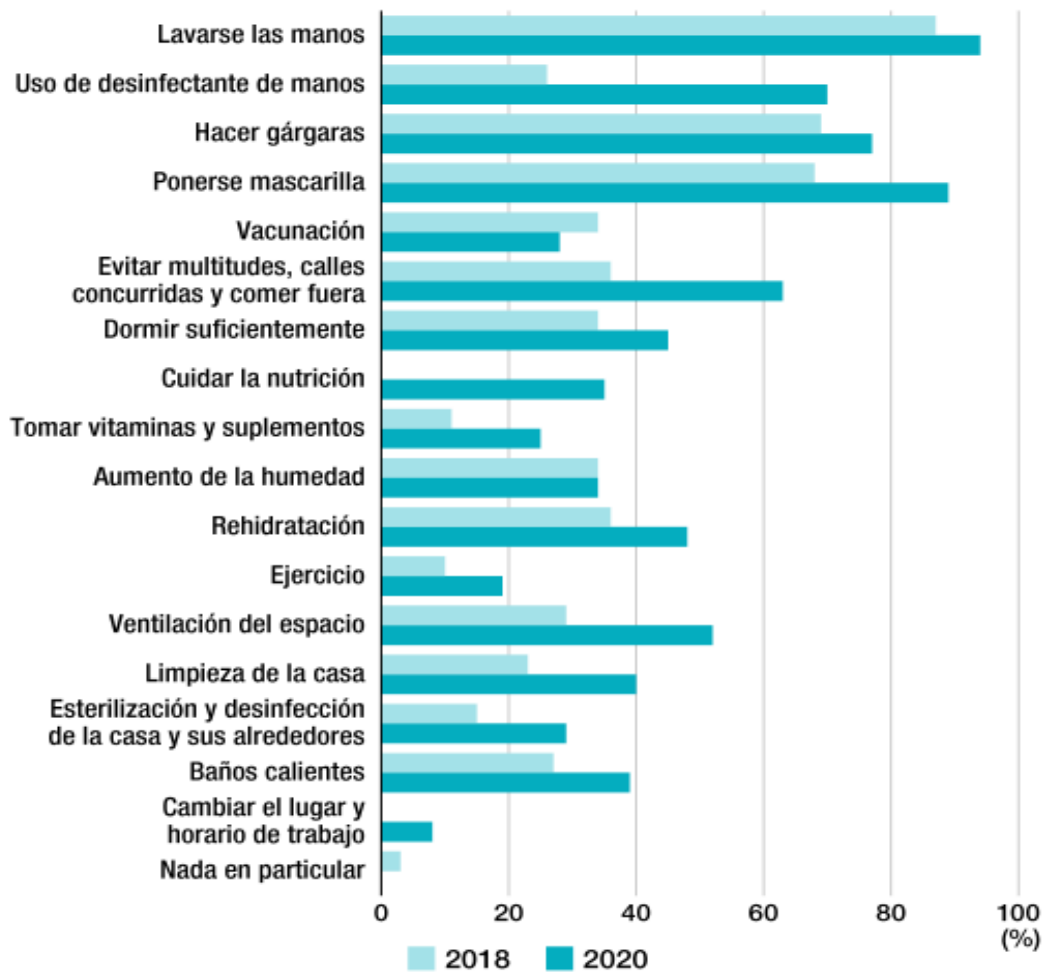

Fuente: Nippon, 2020.

Figura2. Cambios en los hábitos a causa de la nueva pandemia del coronavirus.

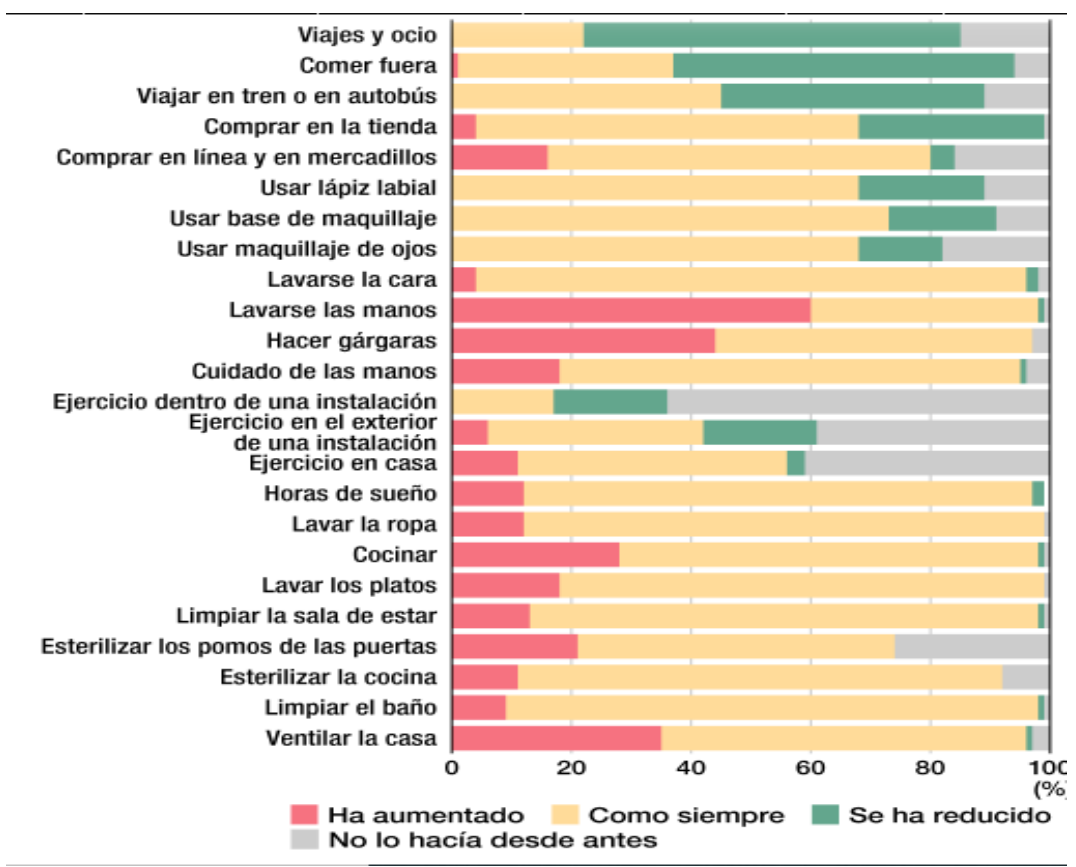

Fuente: Nippon, 2020. 


\section{CONCLUSIONES}

Para finalizar este artículo gracias a toda la investigación pertinentemente hecha se puede decir que se llega al análisis del comportamiento de las personas, al momento de comprar productos de bioseguridad, que en este tiempo se han vuelto un tema básico dentro del comercio y la economía, ya que la mayoría de las personas buscan preservar su vida y su salud cuidándose, de esta forma productos como lo son gel antibacterial, alcohol, entre otros desinfectantes se ven presentes para mitigar un poco el efecto del coronavirus dentro de la población, y a esto se le suma también lo que son los equipos de protección personal que se ven constantemente por necesidad y obligación dentro del medio, como lo son mascarillas que es primordial, guantes, gorros e incluso hasta trajes anti fluidos, y esas podrían ser una de las cosas que más han cambiado dentro nuestra actualidad, y que Por ende influye mucho en el comportamiento de las personas y del mercado en donde se vayan a situar la evolución de nuevos requerimientos, hace que el mercado evoluciones de la mano con estos, Y por supuesto también hay que tener en cuenta que así como la demanda de estos aumentan también se debería de aumentar la calidad específica para estos productos ya que son para evitar enfermedades o propagaciones de las mismas, pero mucho de los consumidores lo que se busca es adquirir y los fabricantes producir, y se deja de un lado las respectivas normalizaciones que se piden a nivel mundial o para su territorio, pero aun así podemos ver que el consumismo no se ve mermado por una pandemia que ha dejado a muchas personas sin sustento o un trabajo fijo, en muchos de los mercados se ve gran afluencia de personas para adquirir productos básicos e incluso los de bioseguridad por supuesto, y es así como la economía y los Estados de comercio dan un giro para poder conseguir un equilibrio, esto de la mano por supuesto con las Industrias, que para preservar una seguridad alimentaria también tiene que ser muy rigurosos al momento de realizar sus procesos o trabajo diario, con planes de retorno al trabajo o de estadía en el trabajo, los cuales tienen que ser Mucho más cuidadosos y pensando en la actual situación del mundo, Por ende todo esto genera un costo que se va a ver reflejado en los nuevos productos no sólo por parte de las Industrias, sino también de los comercios que generan actividades como mercados, centros 
comerciales, entre otros, y es aquí donde encontramos la presencia del comercio por internet o digital, para la adquisición de los mismos, para evitar filas, aglomeraciones y así la propagación del virus, según datos o por simple percepción es muy fácil identificar las diferentes cosas que se han dejado de hacer y otras que sea un mentado cosas simples como lavarse las manos que se vuelven fundamentales en la actualidad y otras que han mermado mucho en su participación para las personas.

\section{REFERENCIAS}

Accenture. (2020). COVID-19 cambiará para siempre el comportamiento de los consumidores. Obtenido de https://www.accenture.com/cles/insights/consumer-goods-services/coronavirus-consumer-behaviorresearch

Alvarado, P. (2020). productos de bioseguridad durante la pandemia. El comercio . Obtenido de https://www.elcomercio.com/actualidad/comprasecuatorianos-bioseguridad-pandemia-coronavirus.html

Angulo, M. (2020). La oscura mina de oro en la que se convirtió la compra de equipos por la pandemia en Latinoamérica. france 24. Obtenido de https://www.france24.com/es/20200508-corrupcion-insumosm\%C3\%A9dicos-covid19-pandemia

Byzness. (2020). El COVID-19 cambia el consumo: menos operaciones, más dinero $y$ supermercados olvidados. Obtenido de https://byzness.elperiodico.com/es/tu-bolsillo/20200422/covid-19consumo-operaciones-dinero-supermercados-7935472

consumo, M. d. (2007 ). PLAN DE CONTINUIDAD DE LAS EMPRESAS FRENTE A EMERGENCIAS, PANDEMIA DE GRIPE. Obtenido de https://www.mscbs.gob.es/ciudadanos/enfLesiones/enfTransmisibles/doc s/EmpresasPlan2.pdf

Daimiel, P. (2020). LOS SEIS COMPORTAMIENTOS DEL CONSUMIDOR ANTE EL COVID-19. Nielsen. Obtenido de https://www.nielsen.com/es/es/insights/article/2020/seiscomportamientos-del-consumidor-ante-covid-19/

expreso. (2020). Las panaderías reinventan su catálogo de productos. Obtenido de https://www.expreso.ec/actualidad/economia/panaderias-reinventancatalogo-productos-12014.html 
Gamarra, G. (2020). Consumidor: perfiles y comportamiento de compra. Soy Marketing. Obtenido de https://soy.marketing/consumidor-perfiles-ycomportamiento-de-c/

GANDINI, G. (2020). ¿Qué hacer con los precios en la pospandemia? Revista. Dinero . Obtenido de https://www.dinero.com/empresas/articulo/comomanejar-los-precios-cuando-acabe-la-pandemia/286867

Julieta. (2020). Aplicación del principio de precaución a la orientación del equipo de protección personal (EPP) durante la pandemia de \#COVID19. Obtenido de https://seguridadbiologica.blogspot.com/2020/07/aplicaciondel-principio-de-precaucion.html

Kantar. (2020). El comportamiento del consumidor en tiempos de coronavirus. Revista enfasis, Alimentacion. Obtenido de http://www.alimentacion.enfasis.com/articulos/85820-el-comportamientodel-consumidor-tiempos-coronavirus

Lemondigital. (2020). ¿Cuál es el comportamiento del consumidor después del COVID-19? Obtenido de https://lemon.digital/comportamiento-delconsumidor-despues-de-pandemia-covid-19/

Meade, G. L. (2020). Los hábitos del consumidor no volverán a ser los mismos después del COVID-19. Ey. Obtenido de https://www.ey.com/es_ec/covid19/habitos-del-consumidor

Méndez, D. (2019). Definición de Comportamiento de compra. Economia Simple. Obtenido de https://www.economiasimple.net/glosario/comportamientode-compra

Nippon. (2020). El uso de maquillaje cae durante la epidemia de la COVID-19. Obtenido de https://www.nippon.com/es/japan-data/h00720/

OMS. (2017). LA GESTIÓN DE RIESGOS ANTE UNA PANDEMIA DE GRIPE. Obtenido de https://apps.who.int/iris/bitstream/handle/10665/272829/WHO-WHE-IHMGIP-2017.1-spa.pdf

PRIETO, M. (2020). Una visión global de cómo está cambiando el comportamiento del consumidor en medio del COVID-19. Smart Lighting. Obtenido de https://smart-lighting.es/comportamiento-consumidor-covid$19 /$

Rico, A. R. (2020). Las 10 tendencias que buscarán los consumidores tras la pandemia del covid-19. La Republica. Obtenido de https://www.larepublica.co/empresas/las-diez-tendencias-que-buscaranlos-consumidores-luego-de-la-pandemia-del-covid-19-2997860 
Social., S. d. (2020). Manual de bioseguridad por motivo de pandemia covid-19 para Centros de Trabajo. Versión 2. Tegucigalpa MDC. Obtenido de http://www.ccichonduras.org/spanish/comunicados/2020/Abril/Comunica dos\%20Web\%20-

\%20Emergencia\%20Covid\%2019/Protocolos\%20Bioseguridad\%20para \%20Centros\%20de\%20Trabajo/Manual\%20de\%20Bioseguridad\%20por \%20motivo\%20de\%20Pandemia\%20COVID-19.pdf

Wallace, A. (2020). Cómo fueron controladas las epidemias de SARS y MERS, otros coronavirus para los que no hay vacuna. BBC News Mundo. Obtenido de https://www.bbc.com/mundo/noticias-52797076 